\title{
Anticoagulación oral
}

\author{
C. DURÁN PARRONDO, C. RODRÍGUEZ MORENO' ${ }^{1}$, F. TATO HERRERO' ${ }^{1}, \mathrm{~N}$. \\ ALONSO VENCE ${ }^{2}$, F. L. LADO LADO ${ }^{3}$ \\ Servicio de Atención Primaria. Lalín. Servicios de ${ }^{1}$ Farmacología Clínica, ${ }^{2}$ Hematología \\ y de ${ }^{3}$ Medicina Interna. Hospital Clínico Universitario. Santiago de Compostela.
}

\author{
ORAL ANTICOAGULATION
}

\section{RESUMEN}

En los últimos años ha aumentado considerablemente la utilización de anticoagulantes orales en la prevención de procesos tromboembólicos. Además de un mayor número de pacientes en tratamiento anticoagulante, actualmente se dispone de una amplísima experiencia sobre las consecuencias médicas y sociales que implica este tratamiento lo que ha originado una visión mucho mas pragmática del manejo cotidiano del paciente anticoagulado. En este artículo se realiza una revisión sobre anticoagulación oral en base a las recomendaciones actuales existentes en la literatura médica.

PALABRAS CLAVE: Anticoagulación oral. Tratamiento antitrombótico. Fibrilación auricular. Prótesis valvular. Warfarina.

\begin{abstract}
The use of oral anticoagulants in the prevention of thrombotics processes, has experienced a considerable increase. In addition, there are a growing experience on the medical and socials consequences of the use of this drug. This has originated a much more pragmatic vision of the daily handling of the anticoagulated patient. In this article, we made a revision about the indications and the practical use, including some useful advices and criteria for the concomitant drug selection.
\end{abstract}

KEY WORDS: Oral anticoagulant. Antithrombotic therapy. Atrial fibrillation. Valvular prostheses. Warfarin.

Durán Parrondo C, Rodríguez Moreno C, Tato Herrero F, Alonso Vence N, Lado Lado FL. Anticoagulación oral. An Med Interna (Madrid) 2003; 20: 377-384.

\section{INTRODUCCIÓN}

En los últimos años numerosos ensayos clínicos (1-4) han demostrado la utilidad de los anticoagulantes orales (ACO) en nuevas indicaciones lo que ha originado un elevado crecimiento en el número de pacientes anticoagulados. Habitualmente, la práctica totalidad de estas prescripciones son realizadas por médicos de atención especializada y tanto los controles periódicos como el seguimiento es llevado a cabo por hematología. No obstante, cada vez es más frecuente que los servicios de atención primaria colaboren con hematología para garantizar un mejor control del tratamiento anticoagulante oral así como facilitar al usuario la realización de las pruebas pertinentes.

En este artículo se revisan las recomendaciones actuales existentes en la literatura médica sobre indicaciones, posología y manejo de la anticoagulación oral, que han variado con el tiempo hacia una actitud mucho más pragmática.

\section{DESCRIPCIÓN DE ANTICOAGULANTES ORALES (ACO)}

Los ACO actúan como antagonistas de la vitamina $\mathrm{K}$, inhibiendo el sistema enzimático encargado de su conversión a la forma activa, que actúa como cofactor en la gamma-carboxilación de los residuos terminales de ácido glutámico de los factores II, VII, IX y X, y de las proteínas C y S; la ausencia de estos residuos ocasiona que estos factores sean inactivos, por la incapacidad de ligar el calcio.

En España el anticoagulante más utilizado es el acenocumarol (Sintrom ${ }^{\circledast}$ en comprimidos de 1 y 4 mg). En los países anglosajones se utiliza la warfarina sódica (Aldocumar ${ }^{\circledR}$ en comprimidos de $1,3,5$ y $10 \mathrm{mg}$ ) y es con este medicamento con el que se han realizado la mayor parte de estudios y ensayos clínicos.

Ambos medicamentos presentan buena absorción oral, elevada unión a proteínas plasmáticas y metabolismo hepático excretándose vía renal Las diferencias fundamentales son farmacocinéticas, con una semivida y una duración de acción menor

Trabajo aceptado: 27 de enero de 2003

Correspondencia: Carmen Durán Parrondo. Servicio de Atención Primaria de Lalín. C/ C, 25. 36500 Lalín. Pontevedra 
para el acenocumarol que para la warfarina (5-9 horas frente a 36-48 horas y 2-3 días frente a 2-5 días, respectivamente) (5-7).

Es importante destacar que existe una gran variabilidad individual en cuanto a la respuesta al tratamiento debido a múltiples factores, tanto farmacocinéticos (absorción, aclaramiento metabólico) como farmacodinámicos (diferente relación concentración/efecto), y a otros como incumplimiento terapéutico, variaciones dietéticas, interacciones farmacólogicas, procesos intercurrentes, etc. También se han descrito casos de resistencia hereditaria o adquirida (5).

\section{INDICACIONES}

Las indicaciones de los ACO (Tabla I) que se revisan a continuación están basadas en las recomendaciones realizadas en el documento de consenso elaborado en la sexta conferencia (año 2000) de terapia antitrombótica del American College

\section{TABLA I}

INDICACIO NES DE LOS ANTICO AGU LANTES O RALES (31)

\begin{tabular}{|c|c|c|}
\hline Indicación & INR & Duración \\
\hline \multicolumn{3}{|l|}{ TVP , TEP: } \\
\hline Secundario a postoperatorio & $2-3$ & 3 meses \\
\hline Esencial & $2-3$ & 6 meses \\
\hline Secundario a causa persistente & & \\
\hline y recidivas & $2-3$ & Indefinido \\
\hline \multirow{2}{*}{\multicolumn{3}{|c|}{$\begin{array}{l}\text { Valvulopatía mitral si: FA crónica } \\
\text { o paroxística, embolismo sistémico }\end{array}$}} \\
\hline & & \\
\hline $\begin{array}{l}\text { previo o aurícula izquierda mayor } \\
\text { de } 55 \mathrm{~mm}\end{array}$ & $2-3$ & Indefinido \\
\hline \multicolumn{3}{|l|}{$\begin{array}{l}\text { Prolapso válvula mitral, si TIA a pesar } \\
\text { de tratamiento con AAS, embolismo } \\
\text { sistémico documentado o FA }\end{array}$} \\
\hline crónica o paroxística & $2-3$ & Indefinido \\
\hline \multicolumn{3}{|l|}{ Valvulopatía aórtica, si embolismo } \\
\hline sistémico previo o FA crónica & $2-3$ & Indefinido \\
\hline \multicolumn{3}{|l|}{ FA sin valvulopatía con factores } \\
\hline de riesgo & $2-3$ & Indefinido \\
\hline \multicolumn{3}{|l|}{ M iocardiopatía dilatada si fracción } \\
\hline \multirow{2}{*}{\multicolumn{3}{|c|}{ IAM anterior extenso si: }} \\
\hline & & \\
\hline \multicolumn{3}{|l|}{ Trombosis mural por ECO-2D } \\
\hline Disfunción severa ventrículo izquierdo & $2-3$ & Indefinido \\
\hline $\begin{array}{l}\text { Bioprótesis valvulares: } \\
\text { Si FA crónica o paroxística, embo- } \\
\text { lismo previo, trombos en aurícula } \\
\text { izquierda o aurícula izquierda }\end{array}$ & $2-3$ & 3 meses \\
\hline$>55 \mathrm{~mm}$ & $2-3$ & Indefinido \\
\hline Prótesis valvulares mecánicas & $2,5-3,5$ & Indefinido \\
\hline $\begin{array}{l}\text { Si embolismo sistémico pese a } \\
\text { tratamiento con ACO con INR } \\
\text { adecuado }\end{array}$ & $\begin{array}{l}\text { Añadir AAS o } \\
\text { aumentar } \\
\text { INR a 2,5-3,5 }\end{array}$ & Indefinido \\
\hline
\end{tabular}

TVP: Trombosis venosa profunda; TEP: Tromboembolismo pulmonar; FA: Fibrilación auricular; TIA: Accidente isquémico transitorio; AAS: Ácido acetilsalicílico; IAM : Infarto agudo de miocardio; ACO : Anticoagulantes orales; INR: Cociente normalizado internacional. of Chest Physicians (ACCP) que es el documento que consideramos de adopción mas universal entre los profesionales involucrados en este tema.

\section{PREVENCIÓN DE TROMBOEMBOLISMO ARTERIAL}

\section{Fibrilación auricular (FA)}

La FA asociada o no a patología valvular es la primera causa de embolismo, con una incidencia media de 6 a 8 por 100 enfermos por año, según existan o no los siguientes factores de riesgo $(8,9,10)$ :

1. Edad $>65$ años, especialmente mujeres $>75$ años.

2. Insuficiencia cardiaca o reducción de la fracción de eyección $(<30 \%)$.

3. Hipertensión no controlada (presión arterial sistólica $>$ $160 \mathrm{mmHg}$ ).

4. Diabetes mellitus.

5. Trombo intraauricular.

6. Embolismo previo.

7. Cardiopatía isquémica.

8. Tirotoxicosis.

-Fibrilación auricular (FA) no reumática: Numerosos ensayos clínicos (11-17) han demostrado la eficacia de la anticoagulación oral en la prevención primaria de accidentes isquémicos en fibrilación auricular no reumática así como que el cociente normalizado internacional (INR), índice que ofrece mejor relación eficacia/seguridad, es el comprendido entre 2 y 3 . En base a estos ensayos clínicos se realizan las siguientes recomendaciones:

- Menores de 65 años y sin factores de riesgo es de elección el ácido acetilsalicílico (AAS) (250-375 mg/día). Si existen factores de riesgo, anticoagular manteniendo el INR entre 2 y 3 .

- Entre 65 y 75 años y sin factores de riesgo se recomienda el AAS (250-375 mg/día) o los ACO (INR entre 2 y 3). Si hay factores de riesgo, son de elección los ACO con INR entre 2 y 3.

- En pacientes > 75 años son de elección los ACO manteniendo un INR entre 2 y 3 . Dado que este grupo presenta mayor riesgo hemorrágico se requieren controles más frecuentes.

Si está contraindicada la ACO se optará por AAS (250$325 \mathrm{mg} /$ día) o si con valores de INR correctos se presenta un episodio tromboembólico, se añade AAS (100mg/día) manteniendo el mismo INR o se aumenta el nivel de anticoagulación $(8,10)$.

-Fibrilación auricular reumática: Los enfermos con FA y estenosis mitral presentan un riesgo embólico 18 veces superior a los individuos en ritmo sinusal y 3 veces mayor que los que presentan FA no reumática. Dada la elevada incidencia de episodios trombóticos en este tipo de pacientes (10 de cada 100/año) se recomienda anticoagular hasta INR comprendido entre 2 y 3, a pesar de la inexistencia de ensayos clínicos en esta situación. Si se produce un embolismo en el curso del tratamiento anticoagulante se recomienda elevar el INR a 2,5-3,5 o añadir AAS 100 mg/día y INR=2-3 $(8,10)$.

-Fibrilación auricular y cardioversión electiva: La cardioversión farmacológica o eléctrica de la FA que persiste más de 48 horas se asocia a una incidencia de embolismo del $5-7 \%$, por lo que se debe anticoagular hasta INR de 2 a 3. Se recomienda iniciar tratamiento 3 semanas antes y mantenerlo 
hasta 4 semanas después de la cardioversión si es el primer episodio de FA, pero si la FA es paroxística se debe mantener el tratamiento según factores de riesgo embólico $(8,10)$.

\section{Prótesis valvulares cardiacas}

En esta situación los ACO han demostrado disminuir significativamente las complicaciones tromboembólicas en mayor cuantía que los antiagregantes por lo que son de elección (1,18-20). Diferentes ensayos clínicos en esta indicación han demostrado que no existe mas eficacia pero sí mayor incidencia de hemorragias cuando el INR sobrepasa el valor de 4,5 por lo que se recomienda mantener un INR por debajo de estos valores $(4,21,22)$.

Las prótesis valvulares mecánicas precisan tratamiento anticoagulante a largo plazo. Durante los tres primeros meses después del recambio se recomienda administrar dicumarínicos manteniendo el INR entre 2,5 y 3,5. Después de los tres meses, se puede reducir a 2-3 en los pacientes con una prótesis aórtica moderna de doble hemidisco sin factores de riesgo asociados $(8,19)$.

La asociación de dosis bajas de AAS (100 mg) con ACO (INR entre 2 y 3 ) reduce aún más los eventos embólicos y mortalidad vascular con un mínimo incremento de complicaciones hemorrágicas. Esta asociación debe considerarse siempre que exista un proceso embólico bajo tratamiento anticoagulante $\mathrm{y}$ en presencia de enfermedad coronaria o vascular periférica $(8,19)$.

Las prótesis valvulares biológicas presentan una menor incidencia de tromboembolismo sin anticoagulación (1-3\% anual), especialmente si el recambio es aórtico (0,5-1\% anual); sin embargo, la incidencia durante los 3 primeros meses es muy superior por lo que se recomienda ACO en este periodo con un INR de 2-3. Si no existen factores de riesgo se recomienda cambiar a los tres meses a AAS (80-325 mg/día) pero, si el paciente tiene historia previa de embolismo, FA crónica o paroxística, presencia de trombo en aurícula derecha, aurícula izquierda dilatada $(55 \mathrm{~mm})$, disfunción ventricular y trombofilia, se recomienda mantener la ACO con un INR entre 2-3 $(8,19)$.

\section{Infarto agudo de miocardio (IAM)}

Los ACO han demostrado prevenir el embolismo sistémico en pacientes de alto riesgo que presenten IAM extenso, especialmente anterior, con función ventricular deprimida, y embolismo previo, FA o trombo mural (23). En estos casos deberán administrarse durante 3 meses postinfarto o indefinidamente si el paciente presenta FA. Se mantiene el INR entre 2 y 3 . En pacientes con bajo riesgo de embolismo son de elección los antiagregantes.

Aunque los ACO han demostrado disminuir el riesgo de reinfarto, muerte y accidente cerebrovascular (ACV) en pacientes que ya han sufrido un infarto, el medicamento de elección para esta prevención secundaria es el AAS por su mejor relación beneficio/riesgo y no requerir controles hematológicos. La asociación de anticoagulantes a dosis bajas (INR=1,4) con AAS no ha demostrado reducir la incidencia de reinfarto, ACV o muerte cardiovascular más que el AAS sólo $(8,24)$.

\section{Prolapso de la válvula mitral}

A pesar de no haber ensayos clínicos que demuestren la eficacia de la anticoagulación oral en esta indicación, se recomienda su utilización en pacientes mayores de 65 años con FA e hipertensión arterial, insuficiencia mitral o insuficiencia cardiaca o en aquellos con ACV previo. También en pacientes con accidente isquémico transitorio recurrente a pesar de estar tratados con AAS. En el resto se recomienda AAS. Se recomienda mantener un INR entre 2-3 (8).

\section{Otros}

En la calcificación de anillo mitral se debe utilizar ACO sólo cuando hay fibrilación o embolismo previo (INR=2-3) $(8,25)$.

En la miocardiopatía dilatada está indicado anticoagular hasta INR entre 2 y 3 si la fracción de eyección es inferior al $30 \%$ y coexiste con fibrilación auricular y tromboembolismo previo o trombo intraventricular. $\mathrm{Si}$ es de origen isquémico o no isquémico en ritmo sinusal y sin factores de riesgo es preferible el AAS (250-325 mg/día) $(8,25)$.

\section{PREVENCIÓN DEL TROMBOEMBOLISMO VENOSO}

\section{En pacientes quirúrgicos}

En pacientes que van a sufrir cirugía general y ortopédica son de elección las heparinas de bajo peso molecular (HBPM). Los ACO están indicados especialmente en pacientes de muy alto riesgo, con un episodio previo de trombosis y/o los que van a sufrir inserción de prótesis de cadera o rodilla. Estos paciente reciben HBPM y además ACO inmediatamente después de la intervención o en el periodo perioperatoiro manteniendo el INR entre 2 y 3 . Se recomienda ACO durante 30 días $(8,26)$.

En enfermos encamados no quirúrgicos se utilizan las HBPM aunque no han demostrado mas eficacia que las heparinas clásicas en disminuir la incidencia de TEP ni la mortalidad. Se indicarán de manera individualizada, en función del riesgo del paciente $(8,26)$.

\section{TRATAMIENTO DE LA TROMBOSIS VENOSA PROFUNDA (TVP) Y EMBOLISMO PULMONAR (TEP)}

La ACO es efectiva en prevenir la trombosis venosa recurrente en pacientes con TVP o TEP. Esta profilaxis secundaria se realiza después del tratamiento trombolítico, comenzando con HBPM y administrando al día siguiente los ACO. Cuando se obtenga el INR adecuado durante dos días consecutivos, se suspende la heparina (alrededor de 4-5 días). No se han observado ventajas clínicamente significativas en prolongar la administración conjunta de heparinas y ACO excepto en el embolismo pulmonar masivo y en la trombosis ileofemoral severa donde se recomienda mantener la heparina durante 10 días (27).

Se aconseja mantener la anticoagulación oral de 6 semanas a 3 meses en pacientes con trombosis distal sintomática o 
con trombosis distal o proximal posterior a cirugía o a una enfermedad médica limitada y de, como mínimo, 6 meses en pacientes con trombosis proximal idiopática. La anticoagulación indefinida estaría indicada en situaciones de trombosis recurrentes idiopática o trombofilia heredada o adquirida (déficit de antitrombina III, de proteínas C, proteína S, fibrilación ventricular de Leiden, etc.). El INR recomendado en todos los casos es entre 2 y $3(27,28)$.

\section{EN QUÉ PACIENTES SON PREFERIBLES LOS ANTIAGREGANTES}

La prevención del tromboembolismo arterial en pacientes con arteriopatía periférica, en bypass aortocoronario, angor estable e inestable y prevención del reinfarto no está indicada mediante anticoagulación oral sino mediante antiagregantes. En caso de angor inestable debe asociarse heparina. En la prevención del accidente isquémico cerebral (ACV) no cardiogénico se recomienda utilizar AAS (29).

Los ACO tampoco son efectivos en la profilaxis secundaria del ictus isquémico cerebral cuando ha fallado la terapia antiagregante. De hecho se ha demostrado (Stroke Prevention in Reversible Isquemic Trial) (30) que, en esta indicación, los ACO inducen un elevado número de complicaciones hemorrágicas mayores, mucho mas numerosas que el AAS.

\section{CONTRAINDICACIONES Y PRECAUCIONES}

La utilización de ACO durante el embarazo se ha asociado con el síndrome warfarínico-fetal, malformaciones del sistema nervioso central (SNC), abortos espontáneos y mortinatos, prematuridad, y hemorragia. El periodo crítico de exposición es entre las 6 y 9 semanas de gestación. Si la madre requiere anticoagulación se optará por heparina o HBPM, al menos entre las semanas 6-12 y a término. También son contraindicaciones absolutas la diátesis hemorrágica, la hemorragia activa y la intolerancia a dicumarínicos.

La contraindicación es relativa en caso de imposibilidad de realizar un tratamiento correcto, hipertensión arterial mal controlada, trastornos de la marcha, hepatopatía severa, aneurisma aórtico o intracraneal, cirugía reciente en el sistema nervioso central u oftalmológica, úlcera péptica o diverticulosis activa, neoplasias urológicas, digestivas o pulmonares.

En caso de pacientes mayores de 75 años existe mayor incidencia de hemorragias por lo que se recomienda comenzar con dosis menor y realizar controles con mayor frecuencia.

También es importante valorar, antes de iniciar el tratamiento, el riesgo individual de hemorragia así como el consumo de alcohol o el incumplimiento terapéutico y tener en cuenta la influencia de factores como: complejos vitamínicos que contengan vitamina $\mathrm{K}$, interacciones con medicamentos, cambios en la dieta y patología concomitante que puede alterar el INR.

\section{EFECTOS ADVERSOS}

La complicación más importante de la anticoagulación oral es la hemorragia. Según distintos estudios (32) el riesgo de sangrado aumenta con el INR (el riesgo aumenta dramáticamente con INR > 4) las patologías asociadas (hipertensión, insuficiencia renal, enfermedad cerebrovascular, etc.), la edad del paciente (mayor riesgo en pacientes mayores de 75 años) o la asociación de determinados medicamentos (AAS, antiinflamatorios no esteroideos-AINEs-).

El riesgo de complicaciones hemorrágicas mayores (p.e. hemorragia intracraneal) es mayor en ancianos, cuando existe historia de ACV o hemorragia gastrointestinal, FA, anemia o insuficiencia renal. No obstante, cuando aparece hemorragia con un INR dentro del rango terapéutico se debe buscar otra causa subyacente.

Es importante destacar que el riesgo de sangrado es mayor durante los primeros meses de tratamiento (32). Otro efecto adverso importante es la necrosis cutánea, la cual suele aparecer entre el tercer y octavo día de comenzar con el tratamiento y se asocia con déficit de proteína $\mathrm{C}$ o $\mathrm{S}$ (más raramente). Además puede aparecer: alopecia, diarrea, náuseas, prurito, urticaria (5).

\section{INTERACCIONES}

Clásicamente se ha dado gran importancia a las interacciones farmacológicas de los anticoagulantes orales con otros medicamentos bien por inducción e inhibición enzimática o por competición por la unión a proteínas plasmáticas o modificaciones de la flora intestinal (5). El enfoque de esta complicación ha variado con el tiempo hacia una actitud actual más sencilla y pragmática. Se pretende perder el miedo a prescribir un medicamento necesario, por la posibilidad de que interaccione con el anticoagulante ( $\mathrm{p}$ ej ciprofloxacino en osteomielitis por gram negativos), siempre que se conozca esta posibilidad, se comunique a hematología y se curse un control a los 2-3 días de comenzar con el tratamiento de manera que la pauta pueda ser ajustada (no olvidar repetir el proceso cuando se retire el nuevo medicamento). Paralelamente se recomienda conocer la existencia de fármacos considerados preferidos para su asociación con ACO y, equivalentes o intercambiables dentro de cada grupo terapéutico, lo que facilita evitar las posibles interacciones; $p$ ej diclofenac como AINE, dipirona y paracetamol como analgésicos menores, pantoprazol como antiulceroso, etc.

Las interacciones más frecuentes e importantes se detallan en la tabla II. Los medicamentos de elección en pacientes anticoagulados se detallan en la tabla III.

Los alimentos ricos vitamina $\mathrm{K}$ como verduras (grelos, espinacas, col, etc.) pueden modificar el INR. Actualmente, lo que se recomienda al paciente es que no modifique su dieta de forma importante (es decir, que no coma durante una semana sólo carne y a la siguiente verdura) pero sin proporcionarle listados de alimentos prohibidos. En caso de que el paciente aumente su ingesta de verduras o modifique su alimentación (por ejemplo dieta para perder peso) se recomienda que se comunique a hematología.

\section{DOSIFICACIÓN}

A pesar de que el efecto anticoagulante se observa a los 23 días de iniciar la ACO, el efecto antitrombótico no se produce hasta que no haya una reducción de los factores II y X (semivida de 40 y 60 horas respectivamente). Por ello, cuando se requiere rapidez en la anticoagulación, se debe administrar simultáneamente HBPM y acenocumarol 1-2 mg/día hasta 
TABLA II

INTERACCIO NES CO N ANTICO AGULANTES O RALES (7)

\section{Potencian anticoagulación}

Alcohol, amiodarona, esteroides anabólicos, cimetidina, clobibrato, cotrimoxazol, eritromicina, fluconazol, isoniazida, metronidazol, miconazol, omeprazol, fenilbutazona, piroxicam, propafenona, propranolol, sulfinpirazona

Paracetamol, ciprofloxacino, dextropropoxifeno, disulfiram, itraconazol, quinidina, fenitoína, tamoxifeno, tetraciclina, vacuna de la gripe.

Cefamandol, cefazolina, gemfibrozilo, heparina, indometacina, sulfisoxazol

AAS, disopiramida, fluorouracilo, ifosfamida, ketoprofeno, lovastatina, ácido nalidíxico, norfloxacino, ofloxacino, sulindaco, tolmetina, salicitalos tópicos

\section{Inhiben anticoagulación}

Barbitúricos, carbamazepina, clordiazepóxido, colestiramina, griseofulvina, nafcilina, sucralfato, alimentos con alto contenido en vitamina K/nutrición enteral,

Dicloxacilina

Azatioprina, ciclosporina, etretinato, trazodona

que disminuyan eficazmente los niveles de protrombina (factor II). Se suspende la heparina cuando se obtenga el INR deseado en dos determinaciones consecutivas continuando con el ACO a la dosis indicada por hematología. Si el tratamiento no es urgente se comienza con acenocumarol 1-2 $\mathrm{mg} /$ día en dosis única y a la misma hora. Dado que la rapidez con la que se alcanza el efecto antitrombótico no depende de la dosis de anticoagulante (es más, elevadas dosis del mismo pueden disminuir la proteína $\mathrm{C}$ aumentando el riesgo de trombosis), no está justificado dar dosis de carga de inicio.

En pacientes con insuficiencia hepática o renal severa, enfermedades concomitantes graves, déficit de vitamina $\mathrm{K}$ desnutrición o ancianos, se puede comenzar con $1 \mathrm{mg} /$ día de acenocumarol.

Durante 1 ó 2 semanas se recomienda realizar controles al tercer y cuarto día, para después espaciar éstos según los resultados, hasta conseguir unos INR adecuados. Se recomienda realizar un control a la semana del alta hospitalaria para valorar la repercusión de los hábitos de vida (actividad física, dieta, consumo de tabaco y alcohol) en el tratamiento.

La duración del tratamiento es indefinida excepto en el infarto por trombosis mural, la bioprótesis valvulares sin otro factor añadido y el TEP y el TEV esencial o secundario a postoperatorio. Los rangos terapéuticos recomendados se cifran en el mantenimiento de un INR entre 2 y 3 excepto en prótesis valvulares mecánicas en los que se recomienda INR entre 2,5 y $3,5(7,34)$.

\section{CONTROL Y SEGUIMIENTO}

El tratamiento ACO requiere un cuidadoso control de su dosificación y una continua vigilancia clínica y analítica. Su monitorización se realiza utilizando el tiempo de protrombina (TP) expresando el resultado en INR o cociente normalizado internacional:

$\mathrm{INR}=(\mathrm{TP}$ plasma del paciente en segundos/TP plasma control en segundos)ISI.

ISI es el índice de sensibilidad internacional de las diferentes tromboplastinas utilizadas como reactivo. El ISI más sensible es de 1 y corresponde a la tromboplastina de referencia internacional. El objetivo de la estandarización del TP es que los resultados sean equivalentes entre diferentes laboratorios y no dependan del reactivo utilizado.

Actualmente existen monitores portátiles que determinan el INR en sangre capilar. Este método presenta la ventaja del control en el domicilio del paciente, disminuyendo el número de visitas al hospital.

Es conveniente realizar un control del INR a los 3-4 días de comenzar con la anticoagulación oral. Una vez conseguido un INR estable y deseado se espaciarán los controles (cada 45 semanas). Si el paciente requiere ajuste de dosis este se hará en función de la dosis total semanal aumentando o disminuyendo entre un 5-20\%. Este cambio no se reflejará en el tiempo de protrombina hasta pasadas al menos 36 horas evaluándose el resultado de las modificaciones hacia los 8 días.

Cuando se introduce un medicamento que potencialmente puede interaccionar con el ACO se debe adelantar el control (a los 2-3 días después de comenzar el nuevo tratamiento) así como si el paciente presenta alguna nueva enfermedad o se sospecha de una reacción adversa debida al anticoagulante.

Se describen a continuación dos pautas de ajuste de dosis: -Pauta 1 (Bridgen y Hirsh) (35):

- INR entre 1,1 y 1,4: Aumentar la dosis total semanal (DTS) un 10-20\% y control semanal.

- NR entre 1,5 y 1,9: Aumentar la DTS un 5-10\% y control en 2 semanas.

- INR entre 3,1 y 3,9: Disminuir la dosis en un 5-10\% y control en 2 semanas.

- INR entre 4 y 6: Suspender acenocumarol el primer día y reducir la DTS un 10-20\% realizando control a la semana.

-Pauta 2. ACCP (34):

- Pacientes con INR > terapéutico pero menor de 5: Suspender anticoagulante y reiniciar a dosis menor cuando esté en rango terapéutico.

- INR entre 5 y 9: si no hay sangrado significativo igual que la categoría anterior. Si hay sangrado, suspender una dosis de acenocumarol y administrar vitamina K (1 a 2,5 mg vía oral). $\mathrm{Si}$ se requiere revertir rápidamente la anticoagulación (p.e: por una urgencia quirúrgica), administrar de 2 a 4 $\mathrm{mg}$ de Vitamina $\mathrm{K}$ vía oral hasta reducción del INR (lo que ocurrirá en 24 horas).

- Pacientes con INR mayor de 9 y menor de 20: suspender anticoagulante, administrar de 3 a $5 \mathrm{mg}$ de vitamina $\mathrm{K}$ vía oral. Monitorizar INR y ajustar dosis (se puede administrar una dosis extra de vitamina $\mathrm{K}$ si es necesario).

- Pacientes con INR mayor de 20 con sangrado severo: suspender anticoagulante y administrar vitamina K, $10 \mathrm{mg}$ por infusión intravenosa lenta, suplementar con plasma fresco o concentrado de complejos de protombina, en función de la urgencia. Se puede repetir administración de vitamina $\mathrm{K}$ cada 12 horas.

En casos no urgentes se recomienda utilizar vitamina $\mathrm{K}$ por vía oral ( en vez de subcutánea), pues numerosos estudios han demostrado que disminuye el INR de manera eficaz y rápida. 
TABLA III

UTILIZACIÓN DE M EDICAM ENTOS EN PACIENTES ANTICO AGULADO S VÍA O RAL (33)

\begin{tabular}{|c|c|}
\hline Antiácidos y antiulcerosos & \\
\hline Almagato, magaldrato & No interaccionan \\
\hline Famotidina & No interacciona \\
\hline Ranitidina & Se acepta su uso \\
\hline O meprazol & $\begin{array}{l}\text { No se considera seguro, en caso necesa- } \\
\text { rio utilizar dosis que no superen los } 20 \\
\text { mg/día y monitorizar }\end{array}$ \\
\hline Pantoprazol & No interacciona \\
\hline Lansoprazol & Se acepta su uso \\
\hline Lactulosa, lactitol, Plantago ovata & No interaccionan \\
\hline Antidiabéticos orales & \\
\hline Sulfonilureas & $\begin{array}{l}\text { Glibenclamida. La unión a proteínas } \\
\text { plasmáticas de las sulfonilureas de } \\
\text { segunda generación (glibenclamida, } \\
\text { glicazida, glimepirida, glipizida, gliqui- } \\
\text { dona,glisentida) es de tipo no iónico y, } \\
\text { por lo tanto, menos susceptible de } \\
\text { interacciones que los antidiabéticos } \\
\text { antiguos }\end{array}$ \\
\hline Acarbosa, miglitol & No interaccionan \\
\hline Rosiglitazona & $\begin{array}{l}\text { Según ficha no se han descrito interac- } \\
\text { ciones, pero por tratarse de un medi- } \\
\text { camento nuevo y con poca experiencia } \\
\text { clínica es necesario precaución. }\end{array}$ \\
\hline Repaglinida, nateglinida & $\begin{array}{l}\text { Según ficha técnica no se han descrito } \\
\text { interacciones, pero por tratarse de } \\
\text { medicamentos nuevos y poca expe- } \\
\text { riencia clínica es necesario precaución }\end{array}$ \\
\hline
\end{tabular}

\begin{tabular}{l}
\hline Antiadrenérgicos de acción periférica \\
Doxazosina, prazosina $\quad$ No interaccionan
\end{tabular}

\section{Diuréticos}

Furosemida, torasemida, No interaccionan

hidroclorotiazida, indapamida

Beta-bloqueantes adrenérgicos
Propanolol
Atenolol

Bloqueantes de canales de calcio

$\begin{array}{ll}\text { Nifedipino } & \text { No interacciona } \\ \text { Verapamilo } & \text { No interacciona } \\ \text { Diltiazem } & \text { Se acepta su uso }\end{array}$

\section{Fármacos activos sobre el sistema renina-angiotensina}

Inhibidores de la enzima conver- Captoprilo, enalaprilo, fosinoprilo,

tidora de angiotensina (IECA) lisinoprilo... No interaccionan

Antagonistas de los receptores de Candesartan, eprosartan, irbesartan,

angiotensina II (ARA II)

losartan, telmisartan, valsartan. No

interaccionan

\section{Antibacterianos, uso sistémico}

Penicilinas amplio espectro
TABLA III (CONT.)

UTILIZACIÓN DE M EDICAM ENTOS EN PACIENTES ANTICO AGULADO S VÍA O RAL (33)

Cefalosporinas

Riesgo de interacción con: moxalactam, cefoperazona, cefamandol, cefminox. Cefonicid ha demostrado ser seguro

M acrólidos Azitromicina se considera seguro. Interaccionan eritromicina, claritromicina y roxitromicina pero su significancia clínica es controvertida

Clindamicina

Aminoglucósidos

Quinolonas

No se han descrito interacciones

Gentamicina no demostró interacción Norfloxacino es relativamente seguro. Se acepta su uso pero se recomienda monitorización

Moxifloxacino y levofloxacino no se han descrito interacciones, pero por tratarse de medicamentos nuevos y poca experiencia clínica es necesario precaución. El resto de quinolonas interaccionan

Fosfomicina

No se han descrito interacciones

\section{Antimicóticos, uso sistémico}

Terbinafina

Se considera seguro. Los derivados imidazólicos (ketoconazol,...) son potentes inhibidores enzimáticos y deben evitarse. Se han descrito interacciones con miconazol vaginal, debido a la absorción a través de mucosas

\section{Mucolíticos}

Acetilcisteína, ambroxol, bromhe- No interaccionan xina, carbocisteína, citiolona

\section{Antitusivos}

Codeína, dextrometorfano, dime- No interaccionan torfano, cloperastina, levodropro-

pizina

Antihistamíncos, uso sistémico
Dexclorfeniramina, cetirizina, No interaccionan
ebastina, astemizol, ketotifeno,
loratadina, mizolastina

\section{0 tros}

Vacuna antigripal

Se han descrito casos de interacción. Se recomienda la inyección subcutánea profunda y monitorizar INR a los 4-5 días de vacunar

O tros medicamentos que se pueden utilizar con seguridad

M etoclopramida, loperamida, digoxina, metildopa, ergotamina, imipramina, levodopa, biperideno, insulina, tolterodina, mebendazol, benzodiazepinas, zolpidem, sumatriptan, zolmitriptan, teofilina, rivastigmina, donezepilo

\begin{tabular}{ll}
\hline $\begin{array}{l}\text { Vitaminas } \\
\text { Vitaminas }\end{array}$ & $\begin{array}{c}\text { Seguras excepto dosis elevadas de vita- } \\
\text { mina E o K }\end{array}$ \\
\hline $\begin{array}{l}\text { Hipolipemiantes } \\
\text { Pravastatina }\end{array}$ & Se considera seguro \\
\hline
\end{tabular}

Amoxicilina, ampicilina, ... se han descrito interacciones por alterar la flora intestinal pero la experiencia clínica hace que se consideren seguros a las dosis habitualmente utilizadas 
TABLA III (CONT.)

UTILIZACIÓN DE M EDICAM ENTOS EN PACIENTES ANTICO AGULADOS VÍA O RAL (33)

\section{Corticoides sistémicos}

Prednisona, metilprednisolona, Se acepta su uso deflazacort

\section{Antiinflamatorios y antirreumáticos}

Diclofenaco, ibuprofeno, naproxeno, nabumetona

Son los AINEs que menos interaccionan. El diclofenaco vía IV presenta mayor riesgo.

Celecoxib y Rofecoxib pueden elevar un $8-10 \%$ el INR.

\begin{tabular}{ll}
\hline $\begin{array}{l}\text { Antigotosos } \\
\text { Colchicina } \\
\text { Alopurinol }\end{array}$ & $\begin{array}{c}\text { No interacciona } \\
\text { Se acepta su uso. Se recomienda moni- } \\
\text { torizar }\end{array}$ \\
\hline Analgésicos & No interacciona \\
Codéna & Se acepta su uso \\
Tramadol & Bajo riesgo de interacción \\
M etamizol & Analgésico de elección. Seguro con 2 \\
Paracetamol & gr/día durante una semana pero inte- \\
& racciona con dosis superiores \\
\hline
\end{tabular}

\section{Antidepresivos}

$\begin{array}{ll}\text { Citalopram } & \text { Se acepta su uso. M onitorizar INR } \\ \text { Sertralina } & \text { Se acepta su uso. } \\ \text { Nefazodona } & \text { Se acepta su uso }\end{array}$

INR: Cociente normalizado internacional; AINEs: Antiinflamatorios no esteroideos; IV: intravenoso.

- Manejo de la ACO durante procesos invasivos (Protocolos del Servicio de Hematología del Hospital Clínico Universitario de Santiago de Compostela):

- Pauta para exodoncia o cirugía menor:

Dos días antes de la extracción o cirugía: a) suspender el acenocumarol que se volverá a reinstaurar de forma crónica el mismo día de la extracción o cirugía; y b) comenzar con
HBPM dosis sc/día por la mañana que se mantendrá hasta el día después de la extracción o cirugía. En caso de exodoncia se recomienda enjuagues con ácido tranexámico o aminocaproico. Las recomendaciones de la ACCP (34) utilizadas para este boletín, no contemplan suspender el anticoagulante oral en caso de exodoncia, salvo elevado riesgo de sangrado

- Pauta para cirugía programada en paciente anticoagulado:

Suspender anticoagulante 3 días antes de la intervención.

Dos días antes de la intervención administrar HBPM sc 40 $\mathrm{mg} / 24$ horas, procurando que la hora de la inyección coincida 12 horas antes de la prevista para cirugía. No es preciso que el paciente ingrese con mayor antelación de la habitual.

Cuando tolere vía oral y no haya riesgo de sangrado reanudar ACO a las dosis previas, comenzando por la más baja y manteniendo Enoxaparina hasta que vuelva a alcanzar anticoagulación terapéutica.

En caso de afectación valvular, realizar profilaxis antibiótica.

Remitir al paciente a hematología para control de anticoagulación dentro de los 15 días siguientes a la intervención.

- Pauta para cirugía programada en anticoagulados de alto riesgo (protesis valvular mecánica):

Suspender acenocumarol 5 días antes de la intervención, ingresando esa tarde en el Hospital.

A la mañana siguiente, iniciar a las 7 horas tratamiento con heparina sódica a dosis de $500 \mathrm{UI} /$ hora, enviando a las 13 horas un tubo con EDTA y un tubo con citrato a hematología para modificar dosis. Se aumenta la dosis de heparina hasta conseguir una TTPA RATIO alrededor de 2.

El día de la intervención se suspenderá heparina 6 horas antes. Dos horas antes de la intervención se administrará calciparina subcutánea 5000 UI/12 horas hasta un total de 3 dosis.

A las 24 horas (72 horas en caso de neurocirugía) de salir de quirófano, reanudar la perfusión de heparina a dosis aproximadamente del $50 \%$ de la que había precisado en el preoperatoio.

En función de situación clínica, drenajes y controles, ir aumentando lentamente dosis de heparina. Cuando tolere la vía oral y no haya riesgo de sangrado reanudar ACO.

\section{Bibliografía}

1. Acar J, Lung B, Boissel JP, Samama MM, Michel PL, Teppe JP, et al. AREVA: multicenter randomized comparison of low-dose versus standard-dose anticoagulation in pacient with mechanical prosthetic Heart valves. Circulation 1996; 94: 2107-2112.

2. Stroke Prevention in Atrial Fibrillation Investigators (SPAF III). Adjusted dose warfarin versus low-intensity, fixed-dose warfarin plus aspirin for high risk patients with atrial fibrillation: Stroke Prevention in Atrial Fibrillation III randomised clinical trial. Lancet 1996; 348: 633-638.

3. Anand SS, Yusuf S. Oral anticoagulant therapy in patients with coronary artery disease: a meta-analysis. JAMA 1999; 282: 2058-2067.

4. Turpie AGG, Gunstensen J, Hirsh, Nelson H, Gent M. Randomized comparison of two intensities of oral anticoagulant therapy after heart valve replacement. Lancet 1988; 1: 1242-1245.

5. Verstraete M, Verhaeghe K, Peerlinck K, Boogaerts MA. Haematological Disorders. En Averery's Drug Treatment. $4^{\mathrm{a}}$ ed. Nueva Zelada 1997; 1113-1163.
6. Whitlon DS, Sadowski JA, Suttie JW. Mechanisms of coumarin action: significance of vitamin K epoxide reductase inhibition. Biochemistry 1978; 17: 1371-1377.

7. Hirsh J, Dalen JE, Anderson DR, Poller L, Bussey H, Ansell J, and Deykin D. Oral anticoagulants: Mechanism of action, clinical effectiveness, and optimal therapeutic range. CHEST 2001; 119: 8S-21S.

8. Heras M, Fernández Ortiz A, Gómez Guindal JA, Iriarte JA, Lidón RM, Pérez gómez F, Roldán I. Guías de actuación clínica de la Sociedad Española de Cardiología. Recomendaciones para el uso del tratamiento antitrombótico en cardiología. Revista Española de Cardiología 1999; 52: 810-820.

9. Atrial Fibrillation Investigators. Risk factors for stroke and efficacy of antithrombotic therapy in atrial fibrillation: análisis of pooled data from five randomized controlled trials. Arch Intern Med 1994; 154: 14491457.

10. Albers GW, Dalen JE, Laupacis A, Manning WJ, Petersen P., Singer 
DE. Antithrombotic Therapy in Atrial Fibrillation. CHEST 2001; 119: 194S-206S.

11. Secondary prevention in non-rheumatic atrial fibrillation after transient ischaemic attack or minor stroke: EAFT (European Atrial Fibrillation Trial) Study Group. Lancet 1993; 342: 1255-1262.

12. Petersen P, Boysen G, Godtfredsen J, Anderson ED, Andersen B. Placebo-controlled, randomised trial of warfarin and aspirin for prevention of thromboembolic complications in chronic atrial fibrillation: the Copenhagen AFASAK study. Lancet 1989; 1: 175-178.

13. Stroke Prevention in Atrial Fibrillation Investigators. Stroke Prevention in Atrial Fibrillation Study: final results. Circulation 1991; 84: 527-539.

14. Boston Area Anticoagulation Trial for Atrial Fibrillation Investigators. The effect of low-dose warfarin on the risk of stroke in patients with nonrheumatic atrial fibrillation. N Engl Med 1990; 323: 1505-1511.

15. Connolly SJ, Laupacis A, Gent M, Roberts RS, Cairns JA, Joiner C. Canadian Atrial Fibrillation Anticoagulation (CAFA) Study. J Am Coll Cardiol 1991; 18: 349-355.

16. Ezekowitz MD, Bridgers SL, James KE, Carliner NH, Colling CL, Gornick CC y cols. Warfarin in the prevention of stroke associated with nonrheumatic atrial fibrillation: Veterans Affairs Stroke Prevention in Nonrheumatic Atrial Fibrillation Investigators. N Engl J Med 1992; 327: 1406-1412.

17. Stroke Prevention in Atrial Fibrillation Investigators. Warfarin versus aspirin for prevention of thromboembolism in atrial fibrillation: Stroke Prevention in Atrial Fibrillation II Study. Lancet 1994; 343: 687-691.

18. Bonow RO, Carabello B, De Leon AC, Edmuns LH, Fedderly BJ, Freed MD y cols. Guidelines for the management of patients with valvular heart disease. Circulation 1998; 98: 1949-1984.

19. Stein P, Alpert JS, Bussey HI, Dalen JE, Turpie AGG. Antithrombotic therapy in patients with mechanical and biological prosthetic heart valves Chest 2001; 119: 220S-227S

20. Mok CK, Boey J, Wang R, Chan TK, Cheung KL, Lee Pk y cols. Warfarin versus dipyridamole-aspirin and pentoxifylline-aspirin for the prevention of prosthetic heart valve thromboembolism: a prospective clinical trial. Circulation 1985; 72: 1059-1063.

21. Saour JN, Sieck JO, Mamo LAR y cols. Trial of different intensities of anticoagulation in patients with prosthetic heart valves. N Engl J Med 1990; 322: 428-432.
22. Altman R, Rouvier J, Gurfinkel E, Scazziota A, Turpie AGG. Comparison of two levels of anticoagulant therapy in patients with substitute heart valves. J Thorac Cardiovasc Surg 1991; 101: 427-431.

23. Cairns JA, Théroux A, Lewis HD, Ezekowitz M, Meade TW. Antithrombotic Agents in Coronary Artery Disease. Chest 2001; 119: 228S$252 \mathrm{~S}$.

24. Smith P, Arnesen H, Holme I. The effect of warfarin on mortality and reinfarction after myocardial infarction. N Engl J Med 1990; 323: 147152.

25. Salem DN, Daudelin DH, Levine HJ, Pauker SG, Eckman MH, Riff J. Antithrombotic therapy in valvular heart disease. CHEST 2001; 119: 207S-219S.

26. Geerrts WH, Heit JA, Clagett GP, Pineo GF, Colwell CW, Anderson FA, Wheeler HB. Prevention of venous thromboembolism. CHEST 2001; 119: 132S-175S.

27. Hyers TM, Agnelli G, Hull RD, Morris TA, Samama M, Tapson V, Weg JG. Antithrombotic therapy for venous thromboembolic disease. CHEST 2001; 119: 176S-193S.

28. Bañas Llanos MH. Nuevas perspectivas en el tratamiento antitrombótico. Inf Ter Sist Nac Salud 2001; 25: 93-104.

29. Albers GW, Amarenco P, Easton JD, Sacco RL, Teal P. Antithrombotic and thrombolytic therapy for ischemic stroke. CHEST 2001; 119: 300S$320 \mathrm{~S}$.

30. The Stroke Prevention in Reversible Ischemia Trial (SPIRIT) Study Group. A randomized trial of anticoagulants versus aspirin after cerebral ischemia of presumed arterial origin. Ann Neurol 1997; 42:857865 .

31. Alonso R, Barroso C, Alvárez I, Puche N, Alcaraz. Anticoagulación oral en atención primaria. Inf Ter Sist Nac Salud 1997; 21: 89-96.

32. Levine MN, Raskob G, Landefeld S, Kearon C. Hemorrhagic complications of anticoagulant treatment. CHEST 2001; 119: 108S-121S.

33. Guerra García MM. Seguridad de medicamentos en pacientes a tratamiento con anticoagulantes orales: acenocumarol. BIM-FARMA 2001 39: $1-4$.

34. Ansell J, Hirsh J, Dalen J, Bussey H, Anderson D, Poller L y cols. Managing oral anticoagulant therapy. CHEST 2001; 119: 22S-38S.

35. Brigden ML. Oral anticoagulant therapy: practical aspects of management. Postgrad Med 1996; 99: 81-102. 